\title{
Acquired perforating dermatosis: association with diabetes and renal failure
}

\author{
Carrie B. Lynde BSc, Melanie D. Pratt MD
}

Previously published at www.cmaj.ca

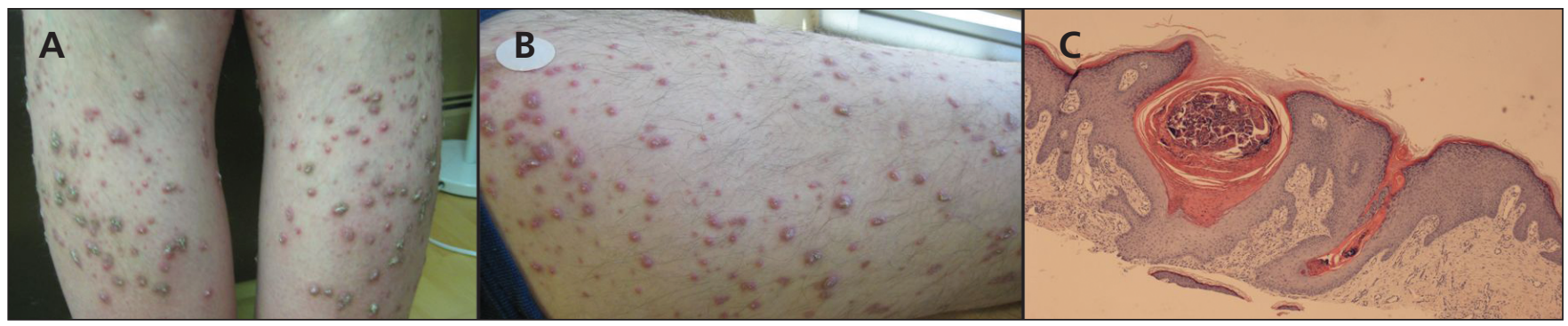

Figure 1: A 46-year-old man with diabetes and renal failure. Follicular keratotic papules consistent with acquired perforating dermatosis were distributed diffusely over his legs (A) and buttocks (B). Skin biopsy (C) showing a hair follicle with perforation extending into the dermal tissue which contains a tract of necrotic tissue debris with degenerate elastin fibres (hematoxylin-eosin stain, original magnification $\times 40$ ).

$\mathrm{A}$ 46-year-old man presented with a pruritic diffuse eruption on his thighs, lower legs and buttocks that had gradually worsened over a 1-year period. His medical history included a heart transplant, insulin-dependent diabetes mellitus, chronic renal failure for which he was receiving peritoneal dialysis, hypertension, gout and hyperlipidemia. An examination showed follicular keratotic papules that were distributed diffusely over his legs and buttocks (Figure 1). The results of a skin biopsy showed a hair follicle with perforation that extended into the dermal tissue and contained a tract of necrotic tissue debris with degenerate elastin fibres (Figure 1). These findings were consistent with acquired perforating dermatosis.

Perforating dermatoses are a group of conditions characterized by transepidermal elimination of dermal material (collagen, elastic tissue or necrotic connective tissue). Major perforating disorders include reactive perforating collagenosis (inherited disorder of collagen perforation), elastosis perforans serpiginosa (elastic tissue perforation associated with Down syndrome and Ehler-Danlos syndrome), perforating folliculitis (perforation of necrotic material secondary to local trauma) and acquired perforating dermatosis. The differential diagnosis includes prurigo nodularis, folliculitis, arthropod bites, multiple keratoacanthomas, psoriasis and lichen planus. ${ }^{1}$

Acquired perforating dermatosis classically presents with severely pruritic follicular hyperkeratotic papules, sometimes

From the Faculty of Medicine (Lynde), University of Toronto, Toronto, Ont., and the Division of Dermatology (Pratt), Department of Medicine, University of Ottawa, Ottawa, Ont.

Cite as CMAJ 2009. DOI:10.1503/cmaj.082013 umbilicated, on the hair-bearing limbs of adults, as in our patient. Generalized papules may also be seen. Acquired perforating dermatosis is a chronic disease, usually associated with diabetes mellitus or renal failure or both. In patients receiving dialysis, acquired perforating dermatosis occurs in about $10 \%$ of patients. ${ }^{2}$ It is also rarely associated with liver disease, malignant disease, hypothyroidism and HIV. ${ }^{3}$

Investigations include skin biopsy and a search for associated systemic diseases. Treatment may be directed at relieving pruritus, because perforating disorders can be exacerbated by koebnerization (lesions appearing at sites of minor trauma, as in psoriasis). Most treatment strategies are supported by anecdotal evidence and include oral or topical retinoids and topical or intralesional corticosteroids. ${ }^{1}$ Phototherapy (narrow-band or broad-band UVB, or psoralen plus UVA) may be helpful for pruritus. Other approaches include the use of antibiotics (e.g., doxycycline) and destructive methods such as cryotherapy, surgical debridement and laser therapy. ${ }^{1}$ Our patient was given retinoids, both oral (acitretin $25 \mathrm{mg}$ daily) and topical (tazarotene gel $0.1 \%$ ). After 4 months of retinoid therapy, the patient's pruritus was substantially reduced and the rash had improved by more than $80 \%$. However, he died suddenly from severe cardiac disease.

\section{REFERENCES}

1. Bolognia JL. Jorizzo JL, Rapini RP, editors. Dermatology. 2nd ed. Spain: Mosby; 2008.

2. Morton CA, Henderson IS, Jones MC, et al. Acquired perforating dermatosis in a British dialysis population. Br J Dermatol 1996;135:671-7.

3. Saray Y, Seckin D. Bilezikci B. Acquired perforating dermatosis: clinicopathological features in twenty-two cases. J Eur Acad Dermatol Venereol 2006;20:679-88. 